\title{
Formal power series in several variables
}

\author{
Pentti Haukkanen \\ Faculty of Information Technology and Communication Sciences \\ FI-33014 Tampere University, Finland \\ e-mail: pentti.haukkanendtuni.fi
}

Received: 11 April 2019

Accepted: 10 October 2019

\begin{abstract}
We present the theory of formal power series in several variables in an elementary way. This is a generalization of Niven's theory of formal power series in one variable. We refer to a formal power series in $n$ variables as an $n$-way array of complex or real numbers and investigate its algebraic properties without analytic tools. We also consider the formal derivative, logarithm and exponential of a formal power series in $n$ variables. Applications to multiplicative arithmetical functions in several variables and cumulants in statistics are presented.
\end{abstract}

Keywords: Formal power series, Derivative, Logarithm, Exponential function, Arithmetical functions in several variables, Cumulants.

2010 Mathematics Subject Classification: 13F25, 11A25, 62E10.

\section{Introduction}

The theory of formal power series is a useful tool in various branches of mathematics. It can be applied, among others, in number theory [1], combinatorics [10], automata theory [5] and statistics [4]. The theory of formal power series can be formulated in various ways. An elementary approach to the theory of formal power series in one variable is presented by Niven [8]. A book on formal power series in one variable is written by Wilf [13].

A formal power series in one variable is an expression of the form

$$
\sum_{i=0}^{\infty} a(i) \theta^{i}
$$

It is in fact an efficient way to present the sequence or the arithmetical function $(a(i))_{i=0}^{\infty}$. The coefficient of $\theta^{i}$ gives the $(i+1)$-st element in the sequence or the value of the arithmetical function at $i$. For example, the formal power series $1+\theta^{2}+\theta^{4}+\cdots$ is an expression of 
the sequence $(1,0,1,0,1, \ldots)$. It can also be written as $1 /\left(1-\theta^{2}\right)$. In the context of formal power series we need not consider the convergence of the power series. We can handle it purely as an algebraic object without analytic tools. Further, we do assign any value to the variable $\theta$. Therefore, it may be referred to as an indeterminate.

The purpose of this paper is to present the theory of formal power series in several variables (or indeterminates) in an elementary way. This theory is a generalization of Niven's theory. We refer to a formal power series in $n$ variables as an $n$-way array of complex or real numbers and investigate its algebraic properties without analytic tools. We also consider the formal derivative, logarithm and exponential of a formal power series in $n$ variables. We give some applications to the theory of multiplicative arithmetical functions in several variables and the theory of cumulants in statistics.

\section{Definition and basic properties}

Definition 2.1. Suppose that $a\left(i_{1}, \ldots, i_{n}\right)$ is a complex number for all $i_{1}, \ldots, i_{n} \in \mathbb{N}_{0}$, the set of nonnegative integers. Then by a formal power series

$$
\sum_{i_{1}, \ldots, i_{n} \geq 0} a\left(i_{1}, \ldots, i_{n}\right) \theta_{1}^{i_{1}} \ldots \theta_{n}^{i_{n}}
$$

in $n$ variables $\theta_{1}, \ldots, \theta_{n}$ we mean the $n$-way array

$$
\left(a\left(i_{1}, \ldots, i_{n}\right): i_{1}, \ldots, i_{n} \in \mathbb{N}_{0}\right) .
$$

We denote by $P_{r}$ the set of formal power series, whose coefficents are real numbers. By $P_{0}$ and $P_{1}$ we mean the sets of formal power series such that $a(0, \ldots, 0)=0$ and $a(0, \ldots, 0)=1$, respectively.

We use the capital letters $A, B, C$ to denote formal power series. The symbols $a\left(i_{1}, \ldots, i_{n}\right)$, $b\left(i_{1}, \ldots, i_{n}\right), c\left(i_{1}, \ldots, i_{n}\right)$ stand for their coefficients.

Definition 2.2. The sum and product of formal power series are defined as

$$
\begin{gathered}
A+B=\sum_{i_{1}, \ldots, i_{n} \geq 0}\left(a\left(i_{1}, \ldots, i_{n}\right)+b\left(i_{1}, \ldots, i_{n}\right)\right) \theta_{1}^{i_{1}} \cdots \theta_{n}^{i_{n}} \\
A B=\sum_{i_{1}, \ldots, i_{n} \geq 0}\left(\sum_{\substack{s_{j}+t_{j}=i_{j} \\
j=1, \ldots, n}} a\left(s_{1}, \ldots, s_{n}\right) b\left(t_{1}, \ldots, t_{n}\right)\right) \theta_{1}^{i_{1}} \cdots \theta_{n}^{i_{n}} .
\end{gathered}
$$

Further,

$$
\begin{aligned}
A=B & \Leftrightarrow \quad a\left(i_{1}, \ldots, i_{n}\right)=b\left(i_{1}, \ldots, i_{n}\right) \quad \text { for all } i_{1}, \ldots, i_{n} \in \mathbb{N}_{0}, \\
A=0 & \Leftrightarrow \quad a\left(i_{1}, \ldots, i_{n}\right)=0 \quad \text { for all } i_{1}, \ldots, i_{n} \in \mathbb{N}_{0}, \\
A=1 & \Leftrightarrow \quad a(0, \ldots, 0)=1, a\left(i_{1}, \ldots, i_{n}\right)=0 \quad \text { otherwise. }
\end{aligned}
$$

Theorem 2.1. The set of formal power series forms an integral domain with respect to the addition and multiplication. 
Proof. We prove only that $A B=0$ implies $A=0$ or $B=0$. Suppose $A B=0$, but $A \neq 0$ and $B \neq 0$. Fix the indices $j_{1}, \ldots, j_{n}$ in the following way: Let $j_{1}$ be the smallest index such that $a\left(j_{1}, i_{2}, \ldots, i_{n}\right) \neq 0$ for some $i_{2}, \ldots, i_{n}$. Let $j_{2}$ be the smallest index such that $a\left(j_{1}, j_{2}, i_{3}, \ldots, i_{n}\right) \neq 0$ for some $i_{3}, \ldots, i_{n}$. Continue in the similar manner. Let us fix the indices $k_{1}, \ldots, k_{n}$ similarly using the coefficents of $B$. Then

$$
\begin{aligned}
\sum_{\substack{s_{i}+t_{i}=j_{i}+k_{i} \\
i=1, \ldots, n}} a\left(s_{1}, \ldots, s_{n}\right) b\left(t_{1}, \ldots, t_{n}\right)= & \sum_{\substack{s_{i}+t_{i}=j_{i}+k_{i} \\
i=2, \ldots, n}} a\left(j_{1}, s_{2}, \ldots, s_{n}\right) b\left(k_{1}, t_{2}, \ldots, t_{n}\right) \\
& \vdots \\
& =a\left(j_{1}, \ldots, j_{n}\right) b\left(k_{1}, \ldots, k_{n}\right) \\
& \neq 0,
\end{aligned}
$$

which is impossible since $A B=0$. This proves that $A=0$ or $B=0$.

\section{Powers}

Definition 3.1. Inverse of a formal power series $A$ is defined by

$$
A A^{-1}=A^{-1} A=1 .
$$

Theorem 3.1. If an inverse of a formal power series exists, it is unique.

Proof. Assume that $A B=B A=1$ and $A C=C A=1$. Then

$$
B=B(A C)=(B A) C=C \text {. }
$$

This proves the theorem.

Theorem 3.2. The inverse of $A$ exists if and only if $a(0, \ldots, 0) \neq 0$.

Proof. Denote $A^{-1}=B$. Then the coefficients of $B$ are determined by the equations

$$
\begin{aligned}
& a(0, \ldots, 0) b(0, \ldots, 0)=1, \\
& a(1,0, \ldots, 0) b(0, \ldots, 0)+a(0, \ldots, 0) b(1,0, \ldots, 0)=0, \\
& \quad \vdots \\
& \sum_{\substack{s_{j}+t_{j}=i_{j} \\
j=1, \ldots, n}} a\left(s_{1}, \ldots, s_{n}\right) b\left(t_{1}, \ldots, t_{n}\right)=0 .
\end{aligned}
$$

This has a solution in $B$ if and only if $a(0, \ldots, 0) \neq 0$.

Definition 3.2. For $m \in \mathbb{N}$, the set positive integers, define

$$
A^{m}=A \cdots A \quad(m \text { factors }) .
$$


Lemma 3.1. Suppose that $B \in P_{1}$. Then $B^{m} \in P_{1}$. Denote $B^{m}=C$. Then

$$
\begin{aligned}
c(1,0, \ldots, 0) & =m b(1,0, \ldots, 0), \\
c(0,1,0, \ldots, 0) & =m b(0,1,0, \ldots, 0), \\
& \vdots \\
c\left(k_{1}, \ldots, k_{n}\right) & =m a\left(k_{1}, \ldots, k_{n}\right)+ \\
& f_{m, k_{1}, \ldots, k_{n}}\left(a\left(i_{1}, \ldots, i_{n}\right): i_{j} \leq k_{j}, j=1,2, \ldots, n ;\left(i_{1}, \ldots, i_{n}\right) \neq\left(k_{1}, \ldots, k_{n}\right)\right)
\end{aligned}
$$

where $f_{m, k_{1}, \ldots, k_{n}}$ is a function of $\left[\left(k_{1}+1\right) \cdots\left(k_{n}+1\right)\right]-1$ variables.

Theorem 3.3. Let $A \in P_{1}$ and $m \in \mathbb{N}$. Then there is a unique $B \in P_{1}$ such that $B^{m}=A$.

Proof. Theorem 3.3 follows easily by Lemma 3.1.

Definition 3.3. Let $A \in P_{1}$ and $m \in \mathbb{N}$. Then the $m$-th root of $A$ is the unique $B \in P_{1}$ such that $B^{m}=A$ and it is denoted as $B=A^{1 / m}$.

Theorem 3.4. Let $a(0, \ldots, 0) \neq 0$ and $m \in \mathbb{N}$. Then

$$
\left(A^{-1}\right)^{m}=\left(A^{m}\right)^{-1} .
$$

Proof. Clearly

$$
A^{m}\left(A^{-1}\right)^{m}=(A A \cdots A)\left(A^{-1} A^{-1} \cdots A^{-1}\right)=1 ;
$$

hence the result holds.

Definition 3.4. Let $A$ be any formal power series. Then define $A^{0}=1$. If $a(0, \ldots, 0) \neq 0$ and $m \in \mathbb{N}$, then define

$$
A^{-m}=\left(A^{m}\right)^{-1} \text {. }
$$

Definition 3.5. Let $m \in \mathbb{Z}, \ell \in \mathbb{N}$ and $A \in P_{1}$. Then the $(m / \ell)$ th power of $A$ is defined as

$$
A^{m / \ell}=\left(A^{1 / \ell}\right)^{m} .
$$

Theorem 3.5. Suppose that $A, B \in P_{r}$ and $m \in \mathbb{N}$. Then, if $m$ is odd, $A^{m}=B^{m}$ implies $A=B$, and if $m$ is even, $A^{m}=B^{m}$ implies $A= \pm B$.

Proof. Clearly it is enough to consider the case $A, B \neq 0$. Suppose that $A^{m}=B^{m}$. Then, $A^{m}-B^{m}=0$ or

$$
\prod_{j=1}^{m}\left(A-\omega^{j} B\right)=0
$$

where $\omega$ is an $n$th root of unity. Therefore

$$
A-\omega^{j} B=0
$$

for some $j=1, \ldots, m$. Since $A, B \neq 0$ and the coefficients of $A$ and $B$ are real numbers, we have

$$
A-\omega^{j} B \neq 0
$$

for all nonreal values of $\omega^{j}$. If $m$ is odd, 1 is the only real number that $\omega^{j}$ can catch up, and if $m$ is even, $\omega^{j}$ can be \pm 1 . Therefore, we have the theorem. 


\section{Derivatives}

Definition 4.1. The derivative of $A$ with respect to the variable $\theta_{k}$ is defined by

$$
D_{k}(A)=\sum_{i_{1}, \ldots, i_{n} \geq 0}\left(i_{k}+1\right) a\left(i_{1}, \ldots, i_{k-1}, i_{k}+1, i_{k+1}, \ldots, i_{n}\right) \theta_{1}^{i_{1}} \ldots \theta_{n}^{i_{n}} .
$$

The $m$-th derivative is defined in the natural way and is denoted by $D_{k}^{m}$.

Definition 4.2. The scalar of $A$ is defined by

$$
S(A)=a(0, \ldots, 0) .
$$

Theorem 4.1. We have

$$
A=\sum_{i_{1}, \ldots, i_{n} \geq 0} \frac{S\left(D_{1}^{i_{1}} \cdots D_{n}^{i_{n}}(A)\right)}{i_{1} ! \cdots i_{n} !} \theta_{1}^{i_{1}} \cdots \theta_{n}^{i_{n}} .
$$

Proof. It can be shown that $S\left(D_{1}^{i_{1}} \cdots D_{n}^{i_{n}}(A)\right)=\left(i_{1} ! \cdots i_{n} !\right) a\left(i_{1}, \ldots, i_{n}\right)$. The theorem follows from this result.

Theorem 4.2. We have

$$
\begin{aligned}
& D_{k}(A+B)=D_{k}(A)+D_{k}(B), \\
& D_{k}(A B)=D_{k}(A) B+A D_{k}(B), \\
& D_{k}\left(A^{m}\right)=m A^{m-1} D_{k}(A), \quad m \in \mathbb{N}, \\
& D_{k}\left(A^{-m}\right)=-m A^{-m-1} D_{k}(A), \quad m \in \mathbb{N} .
\end{aligned}
$$

Proof. We prove only the second statement. The others are evident. Without loss of generality we may assume that $k=1$. Then the general coefficent of the formal power series of the right-hand side is

$$
\begin{aligned}
& \sum_{\substack{s_{j}+t_{j}=i_{j} \\
j=1, \ldots, n}}\left(s_{1}+1\right) a\left(s_{1}+1, s_{2}, \ldots, s_{n}\right) b\left(t_{1}, \ldots, t_{n}\right)+\sum_{\substack{s_{j}+t_{j}=i_{j} \\
j=1, \ldots, n}}\left(t_{1}+1\right) a\left(s_{1}, \ldots, s_{n}\right) b\left(t_{1}+1, t_{2}, \ldots, t_{n}\right) \\
= & \sum_{\substack{s_{1}=1 \\
i_{1}+1}} \sum_{\substack{s_{j}+t_{j}=i_{j} \\
j=2, \ldots, n}} s_{1} a\left(s_{1}, \ldots, s_{n}\right) b\left(i_{1}+1-s_{1}, t_{2}, \ldots, t_{n}\right) \\
& +\sum_{\substack{s_{1}=0 \\
i_{1}}} \sum_{\substack{s_{j}+t_{j}=i_{j} \\
j=2, \ldots, n}}\left(i_{1}+1-s_{1}\right) a\left(s_{1}, \ldots, s_{n}\right) b\left(i_{1}+1-s_{1}, t_{2}, \ldots, t_{n}\right) \\
= & \sum_{s_{1}=1}^{i_{1}} \sum_{\substack{s_{j}+t_{j}=i_{j} \\
j=2, \ldots, n}}\left(i_{1}+1\right) a\left(s_{1}, \ldots, s_{n}\right) b\left(i_{1}+1-s_{1}, t_{2}, \ldots, t_{n}\right) \\
& +\sum_{\substack{s_{j}+t_{j}=i_{j} \\
j=2, \ldots, n}}\left(i_{1}+1\right) a\left(s_{1}, \ldots, s_{n}\right) b\left(0, t_{2}, \ldots, t_{n}\right) \\
& +\sum_{\substack{s_{j}+t_{j}=i_{j} \\
j=2, \ldots, n}}\left(i_{1}+1\right) a\left(0, s_{2}, \ldots, s_{n}\right) b\left(i_{1}+1, t_{2}, \ldots, t_{n}\right) \\
= & \left(i_{1}+1\right) \sum_{s_{j}+t_{j}=i_{1}+1} \sum_{\substack{s_{j}+t_{j}=i_{j} \\
j=2, \ldots, n}} a\left(s_{1}, s_{2}, \ldots, s_{n}\right) b\left(t_{1}, t_{2}, \ldots, t_{n}\right),
\end{aligned}
$$


which is the general coefficient of the formal power series of the left-hand side. Therefore, the statement holds.

Theorem 4.3. Suppose that $A \in P_{1}$ and $r$ is rational number. Then

$$
D_{k}\left(A^{r}\right)=r A^{r-1} D_{k}(A) .
$$

Proof. Denote $r=m / \ell$. Then

$$
D_{k}\left(\left(A^{r}\right)^{\ell}\right)=\ell\left(A^{r}\right)^{\ell-1} D_{k}\left(A^{r}\right)
$$

On the other hand

$$
D_{k}\left(\left(A^{r}\right)^{\ell}\right)=D_{k}\left(A^{m}\right)=m A^{m-1} D_{k}(A) .
$$

Combining the above equations gives the desired result.

Definition 4.3. A sequence $A_{1}, A_{2}, \ldots$ of formal power series admits addition if for each $n$-tuple $\left(r_{1}, \ldots, r_{n}\right)$ there exists a positive integer $N$ such that

$$
a_{j}\left(i_{1}, \ldots, i_{n}\right)=0
$$

for all $j \geq N$ and $0 \leq i_{1} \leq r_{1}, \ldots, 0 \leq i_{n} \leq r_{n}$.

Theorem 4.4. Suppose that $A_{1}, A_{2}, \ldots$ is a sequence admitting addition. Then

$$
D_{k}\left(A_{1}+A_{2}+\cdots\right)=D_{k}\left(A_{1}\right)+D_{k}\left(A_{2}\right)+\cdots
$$

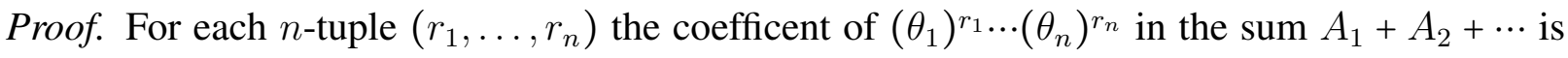
equal to the coefficent of $\left(\theta_{1}\right)^{r_{1} \ldots}\left(\theta_{n}\right)^{r_{n}}$ in the sum $A_{1}+\cdots+A_{N}$. Therefore, the coefficents of

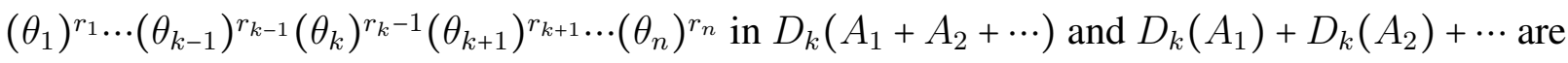
equal. This proves the theorem.

\section{A logarithm function}

Definition 5.1. Let $A \in P_{1}$, and denote $A=1+B$, where $B \in P_{0}$. Then the formal logarithm of $A$ is defined by

$$
\log (A)=\log (1+B)=\sum_{j=1}^{\infty}(-1)^{j+1} \frac{B^{j}}{j} \in P_{0} .
$$

Theorem 5.1. Let $A \in P_{1}$ and $k=1,2, \ldots, n$. Then

$$
D_{k}(\log (A))=A^{-1} D_{k}(A) \text {. }
$$

Proof. By Theorem 4.4,

$$
\begin{aligned}
& D_{k}(\log (A))=D_{k}(\log (1+B))=D_{k}\left(\sum_{j=1}^{\infty}(-1)^{j+1} \frac{B^{j}}{j}\right)=\sum_{j=1}^{\infty} D_{k}\left((-1)^{j+1} \frac{B^{j}}{j}\right) \\
& =\sum_{j=1}^{\infty}(-1)^{j+1} B^{j-1} D_{k}(B)=D_{k}(B) \sum_{j=1}^{\infty}(-1)^{j-1} B^{j-1} \\
& =D_{k}(B)(1+B)^{-1}=D_{k}(A) A^{-1} .
\end{aligned}
$$


Theorem 5.2. If $A, C \in P_{1}$, then

$$
\log (A C)=\log (A)+\log (C) .
$$

Proof. By Theorems 5.1 and 4.2, we obtain

$$
\begin{aligned}
& D_{k}(\log (A C))=(A C)^{-1} D_{k}(A C)=(A C)^{-1}\left[A D_{k}(C)+C D_{k}(A)\right] \\
& =A^{-1} D_{k}(A)+C^{-1} D_{k}(C)=D_{k}(\log (A))+D_{k}(\log (C))=D_{k}(\log (A)+\log (C)) .
\end{aligned}
$$

Since $\log (A C), \log (A)+\log (C) \in P_{0}$, we can deduce the result.

Theorem 5.3. Let $A \in P_{1}$ and let $r$ be a rational number. Then

$$
\log \left(A^{r}\right)=r \log (A)
$$

Proof. By Theorem 5.2, this result holds for positive integers $r$. Since $\log (1)=0$, this result holds for $r=0$. Further, $\log (A)+\log \left(A^{-1}\right)=\log \left(A A^{-1}\right)=\log (1)=0$ and consequently $\log \left(A^{-1}\right)=$ $-\log (A)$. By induction on $r$ we obtain $\log \left(A^{r}\right)=r \log (A)$ for all integers $r$. If $r=m / \ell$, where $m$ and $\ell(\neq 0)$ are integers, we see that $m \log (A)=\log \left(A^{m}\right)=\log \left(\left(A^{r}\right)^{\ell}\right)=\ell \log \left(A^{r}\right)$. Thus $\log \left(A^{r}\right)=(m / \ell) \log (A)=r \log (A)$. Thus the theorem holds.

Theorem 5.4. Let $A, C \in P_{1}$. If $\log (A)=\log (C)$, then $A=C$.

Proof. For any $k=1,2, \ldots, n$,

$$
\left(A C^{-1}\right)^{-1} D_{k}\left(A C^{-1}\right)=D_{k}\left(\log \left(A C^{-1}\right)\right)=D_{k}(\log (A)-\log (C))=D_{k}(0)=0 .
$$

Here

$$
\left(A C^{-1}\right)^{-1} \neq 0
$$

and thus

$$
D_{k}\left(A C^{-1}\right)=0
$$

Therefore

$$
A C^{-1}=1
$$

and so we obtain the theorem.

Theorem 5.5. If $B \in P_{0}$ and $r$ is a rational number, then

$$
(1+B)^{r}=1+r B+\frac{r(r-1)}{2 !} B^{2}+\cdots+\frac{r(r-1) \cdots(r-j+1)}{j !} B^{j}+\cdots
$$

Proof. We adopt the usual notation

$$
\frac{r(r-1) \cdots(r-j+1)}{j !}=\left(\begin{array}{l}
r \\
j
\end{array}\right) .
$$

Let $C$ denote the formal power series of the right-hand side of the equation in the theorem. Then for each $k=1,2, \ldots, n$,

$$
D_{k}(C)=D_{k}(B) \sum_{j=1}^{\infty} j\left(\begin{array}{l}
r \\
j
\end{array}\right) B^{j-1},
$$


and further

$$
\begin{aligned}
(1+B) D_{k}(C) & =D_{k}(B) \sum_{j=1}^{\infty} j\left(\begin{array}{l}
r \\
j
\end{array}\right) B^{j-1}+D_{k}(B) \sum_{j=1}^{\infty} j\left(\begin{array}{l}
r \\
j
\end{array}\right) B^{j} \\
& =D_{k}(B) \sum_{j=1}^{\infty} j\left(\begin{array}{l}
r \\
j
\end{array}\right) B^{j-1}+D_{k}(B) \sum_{j=2}^{\infty}(j-1)\left(\begin{array}{c}
r \\
j-1
\end{array}\right) B^{j-1} \\
& =D_{k}(B) r+D_{k}(B) \sum_{j=2}^{\infty}\left[j\left(\begin{array}{l}
r \\
j
\end{array}\right)+(j-1)\left(\begin{array}{c}
r \\
j-1
\end{array}\right)\right] B^{j-1} \\
& =r D_{k}(B)+D_{k}(B) \sum_{j=2}^{\infty} r\left(\begin{array}{c}
r \\
j-1
\end{array}\right) B^{j-1} \\
& =r D_{k}(B) \sum_{j=0}^{\infty}\left(\begin{array}{l}
r \\
j
\end{array}\right) B^{j}=r D_{k}(B) C
\end{aligned}
$$

Thus

$$
(1+B) D_{k}(C)=r D_{k}(B) C
$$

Multiplying both sides by $C^{-1}(1+B)^{-1}$ we obtain

$$
C^{-1} D_{k}(C)=r(1+B)^{-1} D_{k}(B)=r(1+B)^{-1} D_{k}(1+B)
$$

or

$$
D_{k}(\log C)=D_{k}\left(\log (1+B)^{r}\right)
$$

Since $D_{k}(\log C), D_{k}\left(\log (1+B)^{r}\right) \in P_{0}$, we have

$$
\log C=\log (1+B)^{r}
$$

and thus, by Theorem 5.4,

$$
C=(1+B)^{r}
$$

This completes the proof.

\section{An exponential function}

Definition 6.1. For $B \in P_{0}$ the exponential function is defined by

$$
\exp B=\sum_{j=0}^{\infty} \frac{1}{j !} B^{j} \in P_{1} .
$$

Theorem 6.1. Let $B \in P_{0}$ and $k=1,2, \ldots, n$. Then

$$
D_{k}(\exp B)=D_{k}(B) \exp B .
$$

Proof. Since $\exp B$ admits addition, we have

$$
D_{k}(\exp B)=D_{k}(B) \sum_{j=1}^{\infty} \frac{1}{(j-1) !} B^{n-1}=D_{k}(B) \sum_{j=0}^{\infty} \frac{1}{j !} B^{j}=D_{k}(B) \exp B .
$$


Theorem 6.2. Let $B, C \in P_{0}$. If $\exp B=\exp C$, then $B=C$.

Proof. Let $k=1,2, \ldots, n$. Then

$$
D_{k}(\exp B)=D_{k}(\exp C)
$$

and further

$$
D_{k}(B) \exp B=D_{k}(C) \exp C .
$$

Since $\exp B=\exp C \neq 0$, we have

$$
D_{k}(B)=D_{k}(C)
$$

for all $k=1,2, \ldots, n$. Therefore, $B=C$, which was to prove.

Theorem 6.3. Let $B \in P_{0}$. Then $\log (\exp B)=B$.

Proof. For each $k=1,2, \ldots, n$,

$$
D_{k}(\log (\exp B))=(\exp B)^{-1} D_{k}(\exp B)=(\exp B)^{-1}(\exp B) D_{k}(B)=D_{k}(B) .
$$

Since $B, \log (\exp B) \in P_{0}$, we can conclude the result.

Theorem 6.4. Let $A \in P_{1}$. Then $\exp (\log A)=A$.

Proof. By Theorem 6.3,

$$
\log (\exp (\log A))=\log A .
$$

Thus, by Theorem 5.4, we obtain the result.

Theorem 6.5. Let $B, C \in P_{0}$. Then

$$
\exp (B+C)=(\exp B)(\exp C)
$$

Proof. By Theorems 5.2 and 6.3,

$$
\log ((\exp B)(\exp C))=\log (\exp B)+\log (\exp C)=B+C
$$

Thus, by Theorem 6.4, we obtain the result.

Remark 6.1. It is easy to see that $P_{1}$ forms an Abelian group with respect to multiplication and $P_{0}$ forms an Abelian group with respect to addition. By Theorems 5.4 and 6.2-6.5 we see that these groups are isomorphic.

\section{Applications to multiplicative arithmetical functions}

The theory of multiplicative arithmetical functions of several variables originates to the seminal paper of Vaidyanathaswamy [12] from 1931. Recently, this theory has been developed, e.g., in $[3,6,11]$. Formal power series is a useful tool in this theory as was already noted in [12]. We here review some basics. 
An arithmetical function $f$ of $r$ variables is said to be multiplicative [12] if $f(1, \ldots, 1) \neq 0$ and

$$
f\left(m_{1} n_{1}, \ldots, m_{r} n_{r}\right)=f\left(m_{1}, \ldots, m_{r}\right) f\left(n_{1}, \ldots, n_{r}\right)
$$

for all positive integers $m_{1}, \ldots, m_{r}$ and $n_{1}, \ldots, n_{r}$ with $\left(m_{1} \cdots m_{r}, n_{1} \cdots n_{r}\right)=1$. Clearly, if $f$ is multiplicative, $f(1, \ldots, 1)=1$. A multiplicative function $f$ of $r$ variables is totally determined by the values $f\left(p^{i_{1}}, \ldots, p^{i_{r}}\right)$, where $p$ goes through all primes and $i_{1}, \ldots, i_{r} \geq 0$. This means that it is totally determined by the formal power series

$$
f_{(p)}\left(\theta_{1}, \ldots, \theta_{r}\right)=\sum_{i_{1}, \ldots, i_{n} \geq 0} f\left(p^{i_{1}}, \ldots, p^{i_{r}}\right) \theta_{1}^{i_{1}} \cdots \theta_{r}^{i_{r}}
$$

where $p$ goes through all primes.

An arithmetical function $f$ of $r$ variables is said to be firmly multiplicative [3] if $f(1, \ldots, 1) \neq$ 0 and

$$
f\left(m_{1} n_{1}, \ldots, m_{r} n_{r}\right)=f\left(m_{1}, \ldots, m_{r}\right) f\left(n_{1}, \ldots, n_{r}\right)
$$

for all positive integers $m_{1}, \ldots, m_{r}$ and $n_{1}, \ldots, n_{r}$ with $\left(m_{1}, n_{1}\right)=\cdots=\left(m_{r}, n_{r}\right)=1$. Each firmly multiplicative function is multiplicative. A firmly multiplicative function $f$ of $r$ variables is totally determined by the values

$$
f(1, \ldots, 1, \underbrace{p^{s}}_{j \text { th term }}, 1, \ldots, 1)
$$

where $p$ goes through all primes, $s$ goes through the integers $\geq 1$ and $j$ goes through all the places from 1 to $r$. Each firmly multiplicative function $f$ of $r$ variables can be written in the form $f\left(n_{1}, \ldots, n_{r}\right)=f_{1}\left(n_{1}\right) \cdots f_{r}\left(n_{r}\right)$, where $f_{1}, \ldots, f_{r}$ are multiplicative functions of one variable [11]. Therefore,

$$
f_{(p)}\left(\theta_{1}, \ldots, \theta_{r}\right)=\left(f_{1}\right)_{(p)}\left(\theta_{1}\right) \cdots\left(f_{r}\right)_{(p)}\left(\theta_{r}\right) .
$$

An arithmetical function $f$ of $r$ variables is said to be completely multiplicative (or linear) [12] if $f(1, \ldots, 1) \neq 0$ and

$$
f\left(m_{1} n_{1}, \ldots, m_{r} n_{r}\right)=f\left(m_{1}, \ldots, m_{r}\right) f\left(n_{1}, \ldots, n_{r}\right)
$$

for all positive integers $m_{1}, \ldots, m_{r}$ and $n_{1}, \ldots, n_{r}$. Each completely multiplicative function is firmly multiplicative. A completely multiplicative function $f$ of $r$ variables is totally determined by the values

$$
f(1, \ldots, 1, \underbrace{p}_{j \text { th term }}, 1, \ldots, 1)
$$

where $p$ goes through all primes and $j$ goes through all the places from 1 to $r$. Each completely multiplicative function $f$ of $r$ variables can be written in the form $f\left(n_{1}, \ldots, n_{r}\right)=f_{1}\left(n_{1}\right) \cdots f_{r}\left(n_{r}\right)$, where $f_{1}, \ldots, f_{r}$ are completely multiplicative functions of one variable [11]. Therefore,

$$
f_{(p)}\left(\theta_{1}, \ldots, \theta_{r}\right)=\left(f_{1}\right)_{(p)}\left(\theta_{1}\right) \cdots\left(f_{r}\right)_{(p)}\left(\theta_{r}\right)=\frac{1}{1-f_{1}(p) \theta_{1}} \cdots \frac{1}{1-f_{r}(p) \theta_{r}} .
$$


The Dirichlet convolution of arithmetical functions $f$ and $g$ of $r$ variables is defined as

$$
(f * g)\left(n_{1}, \ldots, n_{r}\right)=\sum_{d_{1} \mid n_{1}} \cdots \sum_{d_{r} \mid n_{r}} f\left(d_{1}, \ldots, d_{r}\right) g\left(n_{1} / d_{1}, \ldots, n_{r} / d_{r}\right)
$$

Let $\delta$ be the arithmetical function of one variable defined as $\delta(1)=1$ and $\delta(n)=0$ otherwise, and let $\delta_{r}$ be the arithmetical function of $r$ variables defined as $\delta_{r}\left(n_{1}, \ldots, n_{r}\right)=\delta\left(n_{1}\right) \cdots \delta\left(n_{r}\right)$. Then $\delta_{r}$ is the identity under the Dirichlet convolution, and it is completely multiplicative. The Dirichlet inverse $f^{-1}$ of an arithmetical function $f$ of $r$ variables exists if and only if $f(1, \ldots, 1) \neq 0$. For each prime $p$, we have

$$
(f * g)_{(p)}\left(\theta_{1}, \ldots, \theta_{r}\right)=f_{(p)}\left(\theta_{1}, \ldots, \theta_{r}\right) g_{(p)}\left(\theta_{1}, \ldots, \theta_{r}\right) .
$$

Further,

$$
\left(\delta_{r}\right)_{(p)}\left(\theta_{1}, \ldots, \theta_{r}\right)=1
$$

and therefore, if $f(1, \ldots, 1) \neq 0$,

$$
\left(f^{-1}\right)_{(p)}\left(\theta_{1}, \ldots, \theta_{r}\right)=\frac{1}{f_{(p)}\left(\theta_{1}, \ldots, \theta_{r}\right)},
$$

If $f$ is a completely multiplicative function of $r$ variables given as $f\left(n_{1}, \ldots, n_{r}\right)=f_{1}\left(n_{1}\right) \cdots f_{r}\left(n_{r}\right)$, where $f_{1}, \ldots, f_{r}$ are completely multiplicative functions of one variable, then

$$
\left(f^{-1}\right)_{(p)}\left(\theta_{1}, \ldots, \theta_{r}\right)=\left(1-f_{1}(p) \theta_{1}\right) \cdots\left(1-f_{r}(p) \theta_{r}\right) .
$$

This means that

$$
f^{-1}\left(n_{1}, \ldots, n_{r}\right)=f_{1}^{-1}\left(n_{1}\right) \cdots f_{r}^{-1}\left(n_{r}\right)=\mu\left(n_{1}\right) f_{1}\left(n_{1}\right) \cdots \mu\left(n_{r}\right) f_{r}\left(n_{r}\right)
$$

where $\mu$ is the number-theoretic Möbius function [1].

\section{Applications to the theory of cumulants}

In this section we apply formal power series to define the cumulants of a random vector and to prove some basic properties for them. A formal point of view has been used previously for example in Speed [9] and Kendall \& Stuart [4]. On the other hand, the cumulants are often defined by the Taylor expansion of the logarithm of the characteristic function (see [7]) and by the Taylor expansion of the logarithm of the moment generating function (see [2]). However, adopting a formal point of view we avoid discussing questions such as convergence and remainders.

Definition 8.1. We define the cumulants $\kappa\left(X_{1}^{\left(r_{1}\right)} \ldots X_{m}^{\left(r_{m}\right)}\right), r_{1}, \ldots, r_{m} \in \mathbb{N}_{0}$, of a random vector $\left(X_{1}, \ldots, X_{m}\right)$ by

$$
\sum_{r_{1}, \ldots, r_{m} \geq 0} \kappa\left(X_{1}^{\left(r_{1}\right)} \ldots X_{m}^{\left(r_{m}\right)}\right) \frac{\theta_{1}^{r_{1}} \cdots \theta_{m}^{r_{m}}}{r_{1} ! \cdots r_{m} !}=\log \sum_{r_{1}, \ldots, r_{m} \geq 0} E\left\{X_{1}^{r_{1}} \cdots X_{m}^{r_{m}}\right\} \frac{\theta_{1}^{r_{1}} \cdots \theta_{m}^{r_{m}}}{r_{1} ! \cdots r_{m} !} .
$$

Remark 8.1. If the expression of a cumulant contains a moment that does not exist, then we define that the cumulant does not exist. 
Theorem 8.1. If the moment $E\left\{X_{1}^{r_{1}} \ldots X_{m}^{r_{m}}\right\}$ exists, then the cumulants $\kappa\left(X_{1}^{\left(s_{1}\right)} \ldots X_{m}^{\left(s_{m}\right)}\right)$ with $s_{1} \leq r_{1}, \ldots, s_{m} \leq r_{m}$ exist.

Remark 8.2. Using the Taylor series Leonov \& Shiryaev ([7], pp. 319-320) noted that if the moments $E\left\{X_{1}^{r_{1}} \ldots X_{m}^{r_{m}}\right\}$ with $r_{1}+\cdots+r_{m} \leq n$ exist, then the cumulants $\kappa\left(X_{1}^{\left(r_{1}\right)} \ldots X_{m}^{\left(r_{m}\right)}\right)$ with $r_{1}+\cdots+r_{m} \leq n$ exist.

Proof. By Definition 8.1, we have

$$
\begin{aligned}
& \sum_{i_{1}, \ldots, i_{m} \geq 0} \kappa\left(X_{1}^{\left(i_{1}\right)} \ldots X_{m}^{\left(i_{m}\right)}\right) \frac{\theta_{1}^{i_{1}} \ldots \theta_{m}^{i_{m}}}{i_{1} ! \cdots i_{m} !}=\log \sum_{i_{1}, \ldots, i_{m} \geq 0} E\left\{X_{1}^{i_{1}} \ldots X_{m}^{i_{m}}\right\} \frac{\theta_{1}^{i_{1}} \cdots \theta_{m}^{i_{m}}}{i_{1} ! \cdots i_{m} !} \\
& =\sum_{\substack{i_{1}, \ldots, i_{m} \geq 0 \\
i_{1}+\cdots+i_{m}>0}} E\left\{X_{1}^{i_{1}} \ldots X_{m}^{i_{m}}\right\} \frac{\theta_{1}^{i_{1}} \cdots \theta_{m}^{i_{m}}}{i_{1} ! \cdots i_{m} !}-\frac{1}{2}\left(\sum_{\substack{i_{1}, \ldots, i_{m} \geq 0 \\
i_{1}+\cdots+i_{m}>0}} E\left\{X_{1}^{i_{1}} \ldots X_{m}^{i_{m}}\right\} \frac{\theta_{1}^{i_{1}} \cdots \theta_{m}^{i_{m}}}{i_{1} ! \cdots i_{m} !}\right)^{2} \\
& +\frac{1}{3}\left(\sum_{\substack{i_{1}, \ldots, i_{m} \geq 0 \\
i_{1}+\cdots+i_{m}>0}} E\left\{X_{1}^{i_{1}} \ldots X_{m}^{i_{m}}\right\} \frac{\theta_{1}^{i_{1}} \cdots \theta_{m}^{i_{m}}}{i_{1} ! \cdots i_{m} !}\right)^{3} \\
& -\frac{1}{4}\left(\sum_{\substack{i_{1}, \ldots, i_{m} \geq 0 \\
i_{1}+\cdots+i_{m}>0}} E\left\{X_{1}^{i_{1}} \ldots X_{m}^{i_{m}}\right\} \frac{\theta_{1}^{i_{1}} \cdots \theta_{m}^{i_{m}}}{i_{1} ! \cdots i_{m} !}\right)^{4}+-\cdots
\end{aligned}
$$

It is easy to see that the coefficent of $\theta_{1}^{s_{1}} \ldots \theta_{n}^{s_{n}}$ consists of terms of the form $E\left\{X_{1}^{i_{1}} \ldots X_{m}^{i_{m}}\right\}$, where $i_{1} \leq s_{1}, \ldots, i_{m} \leq s_{m}$. These moments exist and thus the cumulants $\kappa\left(X_{1}^{\left(s_{1}\right)} \ldots X_{m}^{\left(s_{m}\right)}\right)$ with $s_{1} \leq r_{1}, \ldots, s_{m} \leq r_{m}$ exist. This completes the proof.

Theorem 8.2. Suppose $r_{i j} \in \mathbb{N}_{0}, i \in\{1,2, \ldots, m\}, j \in\left\{1,2, \ldots, n_{i}\right\}$ and $\left(X_{1}, \ldots, X_{m}\right)$ is a random vector such that the moment $E\left\{X_{1}^{r_{11}+\cdots+r_{1 n_{1}}} \cdots X_{m}^{r_{m 1}+\cdots+r_{m n}}\right\}$ exists. Then

$$
\begin{aligned}
& \kappa\left(X_{1}^{\left(r_{11}\right)} \cdots X_{1}^{\left(r_{1 n_{1}}\right)} X_{2}^{\left(r_{21}\right)} \cdots X_{2}^{\left(r_{2 n_{2}}\right)} \cdots X_{m}^{\left(r_{m 1}\right)} \cdots X_{m}^{\left(r_{\left.m n_{m}\right)}\right)}\right) \\
& =\kappa\left(X_{1}^{\left(r_{11}+\cdots+r_{1 n_{1}}\right)} X_{2}^{\left(r_{21}+\cdots+r_{2 n_{2}}\right)} \cdots X_{m}^{\left(r_{m 1}+\cdots+r_{m n}\right)}\right)
\end{aligned}
$$

Proof. As the moment $E\left\{X_{1}^{r_{11}+\cdots+r_{1 n_{1}}} \cdots X_{m}^{r_{m 1}+\cdots+r_{m n}}\right\}$ exists, so by Theorem 8.1 the cumulants in (1) exist. Further, in proving (1) it suffices to consider the case $n_{1}=2, n_{2}=\cdots=n_{m}=1$. Then

$$
\begin{aligned}
& \quad \sum_{r_{11}, r_{12}, r_{2}, \ldots, r_{m} \geq 0} \kappa\left(X_{1}^{\left(r_{11}\right)} X_{1}^{\left(r_{12}\right)} X_{2}^{\left(r_{2}\right)} \ldots X_{m}^{\left(r_{m}\right)}\right) \frac{\theta_{11}^{r_{11}} \theta_{12}^{r_{12}} \theta_{2}^{r_{2}} \cdots \theta_{m}^{r_{m}}}{r_{11} ! r_{12} ! r_{2} ! \cdots r_{m} !} \\
& =\log \sum_{r_{11}, r_{12}, r_{2}, \ldots, r_{m} \geq 0} E\left\{X_{1}^{r_{11}+r_{12}} X_{2}^{r_{2}} \ldots X_{m}^{r_{m}}\right\} \frac{\theta_{11}^{r_{11}} \theta_{12}^{r_{12}} \theta_{2}^{r_{2}} \cdots \theta_{m}^{r_{m}}}{r_{11} ! r_{12} ! r_{2} ! \cdots r_{m} !} \\
& =\log \sum_{r_{1}, r_{2}, \ldots, r_{m} \geq 0} E\left\{X_{1}^{r_{1}} X_{2}^{r_{2}} \ldots X_{m}^{r_{m}}\right\} \frac{\left(\sum_{r_{11}+r_{12}=r_{1}} \frac{r_{1} !}{r_{11} ! r_{12} !} \theta_{11}^{r_{11}} \theta_{12}^{r_{12}}\right) \theta_{2}^{r_{2}} \cdots \theta_{m}^{r_{m}}}{r_{1} ! r_{2} ! \cdots r_{m} !} \\
& =\log \sum_{r_{1}, r_{2}, \ldots, r_{m} \geq 0} E\left\{X_{1}^{r_{1}} X_{2}^{r_{2}} \ldots X_{m}^{r_{m}}\right\} \frac{\left(\theta_{11}+\theta_{12}\right)^{r_{1}} \theta_{2}^{r_{2}} \cdots \theta_{m}^{r_{m}}}{r_{1} ! r_{2} ! \cdots r_{m} !}
\end{aligned}
$$




$$
\begin{aligned}
& =\sum_{r_{1}, r_{2}, \ldots, r_{m} \geq 0} \kappa\left(X_{1}^{\left(r_{1}\right)} X_{2}^{\left(r_{2}\right)} \ldots X_{m}^{\left(r_{m}\right)}\right) \frac{\left(\theta_{11}+\theta_{12}\right)^{r_{1}} \theta_{2}^{r_{2}} \cdots \theta_{m}^{r_{m}}}{r_{1} ! r_{2} ! \cdots r_{m} !} \\
& =\sum_{r_{1}, r_{2}, \ldots, r_{m} \geq 0} \kappa\left(X_{1}^{\left(r_{1}\right)} X_{2}^{\left(r_{2}\right)} \ldots X_{m}^{\left(r_{m}\right)}\right) \frac{\left(\sum_{r_{11}+r_{12}=r_{1}} \frac{r_{1} !}{r_{11} ! r_{12} !} \theta_{11}^{r_{11}} \theta_{12}^{r_{12}}\right) \theta_{2}^{r_{2}} \cdots \theta_{m}^{r_{m}}}{r_{1} ! r_{2} ! \cdots r_{m} !} \\
& =\sum_{r_{11}, r_{12}, r_{2}, \ldots, r_{m} \geq 0} \kappa\left(X_{1}^{\left(r_{11}+r_{12}\right)} X_{2}^{\left(r_{2}\right)} \ldots X_{m}^{\left(r_{m}\right)}\right) \frac{\theta_{11}^{r_{11}} \theta_{12}^{r_{12}} \theta_{2}^{r_{2}} \cdots \theta_{m}^{r_{m}}}{r_{11} ! r_{12} ! r_{2} ! \cdots r_{m} !} .
\end{aligned}
$$

Thus

$$
\begin{aligned}
& \sum_{r_{11}, r_{12}, r_{2}, \ldots, r_{m} \geq 0} \kappa\left(X_{1}^{\left(r_{11}\right)} X_{1}^{\left(r_{12}\right)} X_{2}^{\left(r_{2}\right)} \ldots X_{m}^{\left(r_{m}\right)}\right) \frac{\theta_{11}^{r_{11}} \theta_{12}^{r_{12}} \theta_{2}^{r_{2}} \cdots \theta_{m}^{r_{m}}}{r_{11} ! r_{12} ! r_{2} ! \cdots r_{m} !} \\
= & \sum_{r_{11}, r_{12}, r_{2}, \ldots, r_{m} \geq 0} \kappa\left(X_{1}^{\left(r_{11}+r_{12}\right)} X_{2}^{\left(r_{2}\right)} \ldots X_{m}^{\left(r_{m}\right)}\right) \frac{\theta_{11}^{r_{11}} \theta_{12}^{r_{12}} \theta_{2}^{r_{2}} \cdots \theta_{m}^{r_{m}}}{r_{11} ! r_{12} ! r_{2} ! \cdots r_{m} !} .
\end{aligned}
$$

This gives the result.

Theorem 8.3. Suppose that $\left(X_{1}, \ldots, X_{m}\right)$ is a random vector such that the moments $E\left\{X_{1}^{r_{1}} \ldots X_{k}^{r_{k}}\right\}$ with $k \leq m$ exist. Then

$$
\kappa\left(X_{1}^{\left(r_{1}\right)} \ldots X_{k}^{\left(r_{k}\right)} X_{k+1}^{(0)} \ldots X_{m}^{(0)}\right)=\kappa\left(X_{1}^{\left(r_{1}\right)} X_{2}^{\left(r_{2}\right)} \ldots X_{k}^{\left(r_{k}\right)}\right) .
$$

Proof. By Theorem 8.1 the cumulants in (2) exist. Let

$$
\operatorname{coeff}\left(\frac{\theta_{1}^{r_{1} \cdots \theta_{m}^{r_{m}}}}{r_{1} ! \cdots r_{m} !}\right)[A]
$$

denote the coefficient of

in the formal power series $A$. Then

$$
\frac{\theta_{1}^{r_{1} \cdots \theta_{m}^{r_{m}}}}{r_{1} ! \cdots r_{m} !}
$$

$$
\begin{aligned}
& \kappa\left(X_{1}^{\left(r_{1}\right)} \ldots X_{k}^{\left(r_{k}\right)} X_{k+1}^{(0)} \ldots X_{m}^{(0)}\right) \\
& =\operatorname{coeff}\left(\frac{\theta_{1}^{r_{1}} \cdots \theta_{k}^{r_{k}}}{r_{1} ! \cdots r_{k} !}\right)\left[\log \sum_{i_{1}, \ldots, i_{m} \geq 0} E\left\{X_{1}^{i_{1}} \ldots X_{m}^{i_{m}}\right\} \frac{\theta_{1}^{i_{1}} \cdots \theta_{m}^{i_{m}}}{i_{1} ! \cdots i_{m} !}\right] \\
& =\operatorname{coeff}\left(\frac{\theta_{1}^{r_{1}} \cdots \theta_{k}^{r_{k}}}{r_{1} ! \cdots r_{k} !}\right)\left[\sum_{j=1}^{\infty} \frac{(-1)^{j+1}}{j}\left(\sum_{\substack{i_{1}, \ldots, i_{m} \geq 0 \\
i_{1}+\cdots+i_{m}>0}} E\left\{X_{1}^{i_{1}} \ldots X_{m}^{i_{m}}\right\} \frac{\theta_{1}^{i_{1}} \cdots \theta_{m}^{i_{m}}}{i_{1} ! \cdots i_{m} !}\right)^{j}\right] \\
& =\operatorname{coeff}\left(\frac{\theta_{1}^{r_{1}} \cdots \theta_{k}^{r_{k}}}{r_{1} ! \cdots r_{k} !}\right)\left[\sum _ { j = 1 } ^ { \infty } \frac { ( - 1 ) ^ { j + 1 } } { j } \left(\sum_{\substack{i_{1}, \ldots, i_{k} \geq 0 \\
i_{1}+\cdots+i_{k}>0}} E\left\{X_{1}^{i_{1}} \ldots X_{k}^{i_{k}}\right\} \frac{\theta_{1}^{i_{1}} \cdots \theta_{k}^{i_{k}}}{i_{1} ! \cdots i_{k} !}\right.\right. \\
& \left.\left.+\sum_{\substack{i_{1}, \ldots, i_{m} \geq 0 \\
i_{k+1}+\cdots+i_{m}>0}} E\left\{X_{1}^{i_{1}} \ldots X_{m}^{i_{m}}\right\} \frac{\theta_{1}^{i_{1}} \cdots \theta_{m}^{i_{m}}}{i_{1} ! \cdots i_{m} !}\right)^{j}\right] \\
& =\operatorname{coeff}\left(\frac{\theta_{1}^{r_{1}} \cdots \theta_{k}^{r_{k}}}{r_{1} ! \cdots r_{k} !}\right)\left[\sum_{j=1}^{\infty} \frac{(-1)^{j+1}}{j}\left(\sum_{\substack{i_{1}, \ldots, i_{k} \geq 0 \\
i_{1}+\cdots+i_{k}>0}} E\left\{X_{1}^{i_{1}} \ldots X_{k}^{i_{k}}\right\} \frac{\theta_{1}^{i_{1}} \cdots \theta_{k}^{i_{k}}}{i_{1} ! \cdots i_{k} !}\right)^{j}\right]
\end{aligned}
$$




$$
\begin{aligned}
& =\operatorname{coeff}\left(\frac{\theta_{1}^{r_{1}} \cdots \theta_{k}^{r_{k}}}{r_{1} ! \cdots r_{k} !}\right)\left[\log \sum_{i_{1}, \ldots, i_{k} \geq 0} E\left\{X_{1}^{i_{1}} \ldots X_{k}^{i_{k}}\right\} \frac{\theta_{1}^{i_{1}} \cdots \theta_{k}^{i_{k}}}{i_{1} ! \cdots i_{k} !}\right] \\
& =\kappa\left(X_{1}^{\left(r_{1}\right)} \ldots X_{k}^{\left(r_{k}\right)}\right) .
\end{aligned}
$$

This completes the proof.

\section{References}

[1] Apostol, T. M. (1976). Introduction to Analytic Number Theory, Springer-Verlag, New York.

[2] Brillinger, D. R. (1969). The calculation of cumulants via conditioning, Ann. Inst. Statist. Math., 21, 215-218.

[3] Haukkanen, P. (2018). Derivation of arithmetical functions under the Dirichlet convolution, Int. J. Number Theory, 14 (05), 1257-1264.

[4] Kendall, M. G. \& Stuart, A. (1977). The Advanced Theory of Statistics, Volume 1, 4th edition, Griffin.

[5] Kuich, W. (1997). Semirings and formal power series: Their relevance to formal languages and automata theory. In G. Rozenberg and A. Salomaa, editors, Handbook of Formal Languages, Volume 1, Chapter 9, pages 609-677. Springer-Verlag, Berlin.

[6] Laohakosol, V. \& Tangsupphathawat, P. (2018). An identical equation for arithmetic functions of several variables and applications. Notes on Number Theory and Discrete Mathematics, 24 (4), 11-17.

[7] Leonov, V. P. \& Shiryaev, A. N. (1959). On a methods of calculuation of semi-invariants, Theor. Prob. Appl., 4, 319-329.

[8] Niven, I. (1969). Formal power series, Amer. Math. Monthly, 76, 871-889.

[9] Speed, T. P. (1983). Cumulants and partition lattices, Austral. J. Statist., 25, 378-388.

[10] Stanley, R. P. (1986). Enumerative Combinatorics, Vol. 1, Wadsworth and Brooks/Cole.

[11] Tóth, L. (2014). Multiplicative arithmetic functions of several variables: A survey, in vol. Mathematics Without Boundaries, Surveys in Pure Mathematics, T. M. Rassias, P. M. Pardalos (eds.), Springer-Verlag, pages 483-514.

[12] Vaidyanathaswamy, R. (1931). The theory of multiplicative arithmetic functions, Trans. Amer. Math. Soc., 33, 579-662.

[13] Wilf, H. S. (2005). Generating functionology, AK Peters/CRC Press. 